\title{
A review of the effects of valenced odors on face perception and evaluation
}

\author{
Elmeri Syrjänen ${ }^{1}$, Håkan Fischer ${ }^{1}$, Marco Tullio Liuzza ${ }^{1,2}$, Torun Lindholm ${ }^{1}$, and Jonas K. \\ Olofsson $^{1}$ \\ ${ }^{1}$ Department of Psychology, Stockholm University, Stockholm, Sweden \\ ${ }^{2}$ Department of Surgical and Medical Sciences, "Magna Graecia" University of Catanzaro, \\ Catanzaro, Italy
}

How do valenced odors affect the perception and evaluation of facial expressions? We reviewed 25 studies published from 1995 to 2020 on cross-modal effects between olfaction and facial perception. The results indicate that odors may influence facial evaluations and classifications in several ways. Faces are rated as more arousing during simultaneous odor exposure, and the rated valence of faces is affected in the direction of the odor valence. For facial classification tasks, in general, valenced odors, whether pleasant or unpleasant, decrease facial emotion classification speed. The evidence for valence congruency effects was inconsistent. Few studies found that exposure to a valenced odor facilitates the processing of a similarly valenced facial expression. The results for facial evaluation were mirrored in classical conditioning studies. Faces conditioned with valenced odors were rated in the direction of the odor valence. However, the evidence was inconsistent in facial classification tasks in this literature. Although the research shows some clear patterns, other effects are mixed. Our future research recommendations include greater consideration of individual differences in sensation and cognition, individual differences (e.g., differences in odor sensitivity related to age, gender, or culture), establishing standardized experimental assessments and stimuli, larger study samples, and embracing open research practices.

\section{Introduction}

3 Inferring other peoples' emotional states from their facial expressions is an essential social 34 ability. Historically, facial perception has been studied in isolation; however, social cues are 
1 often ambiguous and rely heavily on contextual information for correct interpretation (Aviezer, 2 Ensenberg, \& Hassin, 2017). How we interpret facial expressions may vary by previous experiences, cultural background, and context (Barrett, Adolphs, Marsella, Martinez, \& Pollak, 2019). In line with this reasoning, several recent reviews have considered the effects of auditory context (Gerdes, Wieser, \& Alpers, 2014), visual cue context, background scene, body posture, situation, culture, and individual differences (Aviezer et al., 2017; Wieser \& Brosch, 2012). However, the contextual effects of odors on facial perception have received less interest. Human olfactory abilities are capable of detecting environmental odors even at minute concentrations (McGann, 2017), but olfactory perception needs visual confirmation to establish its identity or social relevance (Olofsson \& Gottfried, 2015). This research review aims to integrate the emerging insights from how odors are perceptually integrated with emotionally expressive face stimuli.

Olfaction provides an interesting vantage point for studying cross-sensory integration of emotional cues. Olfaction has been described as an inherently emotionally evocative sense, presumably because of its close integration with the limbic system (Soudry, Lemogne, Malinvaud, Consoli, \& Bonfils, 2011). Olfaction is involved in detecting and identifying health hazards (e.g., fire, bad food) and food and nutrition (de Vries et al., 2020; Stevenson, 2010). This link between olfaction and survival might explain the emotional nature of the olfactory system. For example, valenced odors can elicit basic emotions, most notably happiness and disgust (Alaoui-Ismaïli, Robin, Rada, Dittmar, \& Vernet-Maury, 1997; Croy, Olgun, \& Joraschky, 2011; Glass, Lingg, \& Heuberger, 2014). Thus, odors have profound emotional and motivational effects on human behavior.

Due to the close link between odors and emotions, odors might affect the processing of social cues. There is a broad agreement that olfaction's main hedonic dimensions are valence and arousal (Anderson et al., 2003; Bensafi, 2002). Valenced odors might, perhaps unsurprisingly, affect mood states such that pleasant odors have a positive effect. In contrast, unpleasant odors have a negative effect on mood (Herz, 2009), and odors may also be experienced as energizing, sensual, or disgusting (Ferdenzi et al., 2011). In general, the arousing properties of odors may facilitate the overall processing of stimuli. Corroborating this notion, (Millot, Brand, \& Morand, 2002) found faster reaction times (RTs) to simple visual and auditory cues in both unpleasant and pleasant odor contexts compared to a no-odor context. Apart from the general arousing properties of odors, there is evidence of odor-visual integration from research on object 
1 congruency. Smelling an orange can increase visual attention towards oranges (Seo, Roidl, 2 Muller, \& Negoias, 2010). Behavioral studies also show that congruent cues from olfactory and visual objects are processed in parallel in the early stages of stimulus processing, but they are combined at later stages to benefit behavioral decisions (Amsellem, Hochenberger, \& Ohla, 2018). Thus, there are several ways by which odors might influence facial perception, including arousal and valence effects, as well as their interactions with facial expressions that are congruent or incongruent with the emotion elicited by the odor.

\section{In ecological settings, perception is often multisensory. Previous research has shown that when} we use congruent cues from multiple modalities, our ability to recognize objects becomes faster and more accurate. For example, in a noisy environment, seeing a speaker's lips will improve the ability to hear the verbal content (Ross, Saint-Amour, Leavitt, Javitt, \& Foxe, 2007). Odors can, in a similar manner as hearing, influence visual perception. Most research on the influence of odors on perception has focused on matching congruent vs. incongruent object cues (e.g., the smell and picture of an orange), presumably because such matching corresponds to the processing demands of widely used odor identification tests (Doty, Smith, McKeown, \& Raj, 1994; Hummel, Sekinger, Wolf, Pauli, \& Kobal, 1997; Olofsson \& Gottfried, 2015). Here we focus instead on the relation between odor and perception of facial expressions. Effectively integrating odor and face cues might help evaluate the source and potential health hazards associated with the odor. However, the emotional properties of odors might also lead to biased evaluations of face stimuli. The current review thus focuses on how odors affect the perception of faces.

The basic emotion theory (Ekman, Sorenson, \& Friesen, 1969) posits six universal basic emotions (happiness, fear, disgust, anger, surprise, and sadness), each associated with distinct facial expressions, physiological changes, and behavioral patterns. However, many of these assumptions, such as the universality (Russell, 1993), facial expressions (Barrett et al., 2019) and physiological changes (Lindquist, Wager, Kober, Bliss-Moreau, \& Barrett, 2012) of the basic emotion theory have been questioned. As noted above, recent theoretical work has emphasized the contextual effects in emotions (Barrett, 2017a). In this view, in emotion perception, all available information is used to predict and correct our model of the world using active inference such as past experiences, culturally appropriate displays, and contextual information in our environment (Barrett, 2017b). The last 25 years have seen a steady increase in research on contextual effects on facial perception. In our current review, we retain the 
1 classification of emotional expressions provided by the basic emotion theory. However, we

2 highlight the importance of how an olfactory context affects facial perception and associated 3 behaviors. The behavioral measures comprise affective ratings, emotion classification 4 accuracy, and response times (RTs) in various tasks.

5

\section{Method}

\section{Results}

We performed searches in Google Scholar, PsycINFO, PubMed, and Web of Science for keywords: (face, odor, smell, olfaction \& cross-modal), as well as in our databases of articles, with the last search conducted on September 1, 2020. Articles matching our criteria were also manually searched in the reference lists of all included articles. We did not include studies attempting to study human pheromones or chemosignals; as this research is based on assumptions of unique properties of certain chemosensory stimuli, these reports fell outside of our review's scope to integrate research findings across different stimuli and experimental settings whenever possible. We included articles that fulfilled the following inclusion criteria.

\section{Peer-reviewed empirical studies available in English}

2. Healthy adult participants

3. Reported behavioral effects of odors on the perception of faces

In total, we found 25 articles matching the inclusion criteria; these articles were published from 1995 until September 2020 and are presented with summaries in Table 1. The table comprises the studies listed in chronological order, an overall description of the design, stimuli, participants, and main findings.

An overview of the included studies allows for some general observations regarding the most commonly used methods and stimuli. The methods can be roughly divided into three experimental paradigms. First, facial emotion classification tests where RT and accuracy levels were measured. Second, subjective evaluations (i.e., ratings of facial valence and arousal) during exposure to various odors. Third, classical conditioning studies where neutral faces were associated with valenced odors; these studies used both emotion classification tasks and facial evaluations as outcome measures. Several studies included electrophysiological measures or 
1 functional magnetic resonance imaging (fMRI); however, here, we focus on behavioral effects.

2 Odor stimuli were typically either clearly pleasant (vanillin and floral-based, such as jasmine)

3 or unpleasant (valeric acid, hydrosulfide, methyl mercaptan, and fish). A majority of the

4 included studies also used clean air as a no-odor control condition; however, few studies used

5 emotionally neutral odors as a control. Common facial expressions used in the reviewed studies

6 were happy, neutral (most prevalent in associative learning studies), disgusted, fearful/anxious,

7 and angry expressions.

Table 1: Description and main results of behavioral studies of face perception during odor exposure, studies listed chronologically.

\begin{tabular}{|c|c|c|c|c|c|}
\hline & & Odor delivery & & $\mathbf{N}$ & \\
\hline Study (year) & Design & $\begin{array}{l}\text { Event/Blocked } \\
\text { (E/B) }\end{array}$ & Odor/Face (O/F) & $\begin{array}{l}\text { Female/male } \\
(\mathbf{F} / \mathbf{M})\end{array}$ & Main findings \\
\hline
\end{tabular}

\begin{tabular}{|c|c|c|c|c|c|}
\hline \multirow{5}{*}{$\begin{array}{l}\text { Todrank. } \\
\text { Byrnes, et al. } \\
(1995)\end{array}$} & Liking of neu faces & \multirow{5}{*}{ E } & & & \\
\hline & paired with ple, neu, or & & \multirow{4}{*}{$\begin{array}{l}\text { O: Ple, neu, unp } \\
\text { F: Neu }\end{array}$} & Exp 1: $20(15 \mathrm{~F})$ & Faces paired with people-related odors were liked in \\
\hline & unp odors. Exp 1, 2, 4 & & & $\operatorname{Exp} 2: 15(11 \mathrm{~F})$ & the direction of odor valence in $\operatorname{Exp} 1,2 \& 4$. This \\
\hline & people-related odors. & & & Exp 3: 14 (9 F) & effect was not evident in Exp 3, where object-related \\
\hline & Exp 3, object-related & & & Exp 4: $23(16 \mathrm{~F})$ & odors were used. \\
\hline
\end{tabular}

Attractiveness of

Cann \& Ross (1989)

attractive and

unattractive female faces $B$

during exposure to ple,

no-odor, or unp odors.
O: Ple, no-odor, unp

F: Neu

$63 \mathrm{M}$

Odors did not affect attractiveness ratings
Val and arousal ratings

Hermann,

Ziegler,

Birbaumer, and

Flor (2000)

Bensafi,

Pierson, et al.

(2002)

Gottfried,

O'Doherty, and

Dolan (2002)

\section{(SAM-scale) of neu}

faces conditioned by

startle sounds during

E

exposure to ple $(n=15)$ or

unp odor $(n=15)$.

Neutral faces classified

as ple or unp during

exposure to ple odor or

E

no-odor.

Neu faces paired with

ple, no-odor, or unp

odor. RTs obtained in $\mathrm{E}$

face gender classification

task.
O: Ple, no-odor

F: Neu

$15 \mathrm{~F}$

Plea odor did not affect face classification.
Faces paired with a startle sound during unp odor exposure were rated as more arousing and neg than other conditions. Faces paired with a startle sound during a ple odor exposure were rated as more arousing but not more pos.

Slower RTs for faces conditioned to ple and unp odors

O: Ple, no-odor, unp

F: Neu

$17(10 \mathrm{~F})$ in the first block, no-effect in the second and third block. 
Leppanen and

Hietanen

(2003)

Gottfried and

Dolan (2004)

Osterbauer, and Spence

(2007)

Li, Moallem,

Paller, and

Gottfried

(2007)

\section{Seubert,}

Loughead, et

al. (2010)
Exp 1. Face emotion

classification task (happy

or disgusted) during ple

or unp odor exposure.

Exp 2. Similar to 1 but

with a between-groups

design, added neu faces,

and no-odor controls.

$2 \mathrm{CS}+$ neutral faces

paired with UCS odors, 2

faces CS- not paired with

an unp odor. RTs for E

gender identification,

faces were rated (VAS)

on intensity and valence.

\section{Women rated (VAS)}

attractiveness of male

faces during exposure to

ple and unp odors.

Likeability of neu faces

rated (VAS) after

exposure to sub-

E

threshold ple, neu, no-

odor, and unp odors.

Emotion classification

task (happy, neu,

disgusted faces) in event- $E$

related design with ple,

no-odor, and unp odors.

\section{Emotion classification}

Seubert,

Kellermann, et

al. (2010)

Steinberg et al

(2012)

Face valence and arousal

ratings (SAM) to $\mathrm{CS}+\mathrm{E}$

(unp odor) and CS- faces

Preference for neu faces

Steinberg et al. when previously paired

(2013)
Exp 1.

O: Ple, unp

F: Happy, Disgusted

Exp 2.

O: Ple, no-odor, unp

F: Happy, neu,

disgusted

O: Unp

F: Neu

$18(10 \mathrm{~F})$

Exp 1: $20(15 \mathrm{~F})$

$\operatorname{Exp} 2: 45(40 \mathrm{~F})$

$\mathrm{F}$ : Neu (varying in $16 \mathrm{~F}$

attractiveness)

O: Ple, neu, no-odor,

unp

$31(18 \mathrm{~F})$

F: Neu

O: Ple, no-odor, unp

F: Happy, neu,

$24(10 \mathrm{~F})$

disgusted

O: Ple, no-odor, unp

F: Happy, neu,

disgusted

O: Unp, no-odor

F: Neu

O: Ple, no-odor, unp

F: Neu

$23(12 \mathrm{~F})$ context, slower in no-odor control, and slowest in unp odor condition.

Faster RTs to CS+ first half of the exp. CS+ faces were rated as more neg val compared to CS-.

Faces rated as less attractive during exposure to unp odor.

$\mathrm{CS}+$ faces rated as more negative post conditioning, no arousal effects.

Exp 1. Happy faces recognized faster in ple odor context; effect was absent in unp odor condition, where disgusted faces were recognized slightly faster. Exp 2. Happy faces recognized faster in ple odor

Odor-unaware subgroup $(\mathrm{n}=15)$ rated faces as less likable after unp than after ple odor.

Faster and more accurate classification of disgust in ple and unp odor condition than in neutral odor condition. Response times to happy faces increased in ple and unp odor conditions compared to neu odor.

Faster classification of disgust in ple and unp odor

Preference for face depended on associated odor (pleasant $>$ neutral $>$ unpleasant).

odors. 


Seubert,
Gregory,
Chamberland,
Dessirier, and
Lundstrom
(2014)

Cook et al.

(2015)

Leleu, Demily,

et al. (2015)
O: Ple, unp odor mixtures controlling for intensity (linearly increasing in val from ple to unp). $\mathrm{F}$ : Neu (varying in attractiveness and age)

O: Ple, no-odor, unp F: Neu no-odor, or an unp odor

\section{Matching faces with} varying levels of emotional expressions during exposure to ple, no-odor, and unp odor.

\section{Val ratings (VAS) and}

emotion classification of

Novak et al.

(2015) neu and fearful faces during neu or subthreshold unp odor exposure.

Val ratings (VAS) of

Cook et al. (2017)

\section{neutral faces during} exposure to a ple, unp, or neu odor.

Syrjänen et al.

$$
\text { od }
$$

RTs and accuracy in

emotion classification

task of changing faces.

\section{Detection of facial}

Damjanovic, emotion in a visual
O: Ple, no-odor, unp

F: Happy, angry,

fearful \& sad

(morphed with neu

for emotion levels)

O: Neu, unp (diluted

with neu odors below

F: Neu (morphed

with fearful faces in

$2 \%$ or $12 \%$ )

O: Ple, no-odor, unp

F: Happy, disgusted

O: Ple, no-odor, unp

F: Happy, neu,

$21(13 \mathrm{~F})$

disgusted

O: Ple, no-odor, unp

F: Happy, neu, angry detection threshold)

$23(13 \mathrm{~F})$
Faces were rated as more attractive and younger in ple compared to unp odor condition.

Ratings of faces were affected by odor priming in the direction of odor val.

Congruent odors increased sensitivity for face emotion matching (e.g., happy faces in the ple odor condition) for happy, disgusted, and angry expressions. Fewer false alarms for disgusted faces in the unp odor condition when emotion labels for faces were provided. Without labels, more false alarms were provided for neg emotions in the ple odor context.

No effects of odors on rated val or emotion recognition accuracy

Happy faces were rated more ple during exposure to a ple odor than neu or unp odor; disgusted faces rated as less ple during exposure to unp odor compared to plea and neu odor.

Odors did not affect ratings of face valence or arousal. Faster classification of faces in unp odor condition. Classification accuracy was not affected by odors.

Faster detection of happy faces in neu and unp odor conditions. Similar effect for ple odor early in the experiment, but effect decreased over time. In contrast, unp odor made detection of happy faces faster later in the experiment. 


\begin{tabular}{|c|c|c|c|c|c|}
\hline $\begin{array}{l}\text { Cook et al. } \\
(2018)\end{array}$ & $\begin{array}{l}\text { Valence ratings of } \\
\text { neutral faces during, or } \\
\text { one second after } \\
\text { exposure to a ple, no- } \\
\text { odor, or unp odor. }\end{array}$ & E & $\begin{array}{l}\text { O: Ple, no-odor, unp } \\
\text { F: Neu }\end{array}$ & $28(18 \mathrm{~F})$ & $\begin{array}{l}\text { Faces rated less ple when primed with unp odor; effect } \\
\text { largest when odor and faces were presented } \\
\text { concurrently. }\end{array}$ \\
\hline $\begin{array}{l}\text { Syrjänen et al. } \\
\text { (2018) }\end{array}$ & $\begin{array}{l}\text { Val and arousal ratings } \\
\text { (SAM) of facial } \\
\text { expressions during } \\
\text { exposure to unp, ple, and } \\
\text { no-odor }\end{array}$ & B & $\begin{array}{l}\text { O: Ple, no-odor, unp } \\
\text { F: Happy, neu, } \\
\text { disgusted }\end{array}$ & 58 (33 female) & $\begin{array}{l}\text { Faces rated more neg during unp odor exposure. } \\
\text { Happy faces were rated as less arousing in unp than } \\
\text { ple odor condition. }\end{array}$ \\
\hline $\begin{array}{l}\text { Syrjänen et al. } \\
(2019)\end{array}$ & $\begin{array}{l}\text { Val and arousal ratings } \\
\text { (SAM) of facial } \\
\text { expressions during } \\
\text { exposure to unp, ple, and } \\
\text { no-odor }\end{array}$ & B & $\begin{array}{l}\text { O: Ple, no-odor, unp } \\
\text { F: Happy, neu, } \\
\text { disgusted }\end{array}$ & $40(22 \mathrm{~F})$ & $\begin{array}{l}\text { More pos ratings in the ple odor and more neg ratings } \\
\text { in the unp odor exposure. Higher arousal ratings } \\
\text { during odor exposure vs. no-odor. }\end{array}$ \\
\hline Li et al. (2020) & $\begin{array}{l}\text { Emotion classification } \\
\text { task on happy, neu, and } \\
\text { fearful faces during } \\
\text { exposure to ple, neu, or } \\
\text { unp odors. }\end{array}$ & B & $\begin{array}{l}\text { O: Ple, no-odor, unp } \\
\text { F: Happy, neu, } \\
\text { fearful }\end{array}$ & $54(28 \mathrm{~F})$ & $\begin{array}{l}\text { Higher accuracy during unp odor exposure. Faster } \\
\text { classification in unp odor condition. Effect was } \\
\text { pronounced for fearful faces. }\end{array}$ \\
\hline $\begin{array}{l}\text { Stankovic et al. } \\
(2020)\end{array}$ & $\begin{array}{l}\text { Binary face classification } \\
\text { task (emotion vs. neutral) } \\
\text { with stimuli in right or } \\
\text { left visual field. } \\
\text { Breathing through either } \\
\text { left or right nostril during } \\
\text { unp odor exposure. }\end{array}$ & B & $\begin{array}{l}\text { O: Unp } \\
\text { F: Neu, happy, } \\
\text { surprised, fearful, } \\
\text { angry, disgusted, sad }\end{array}$ & $60 \mathrm{~F}$ & $\begin{array}{l}\text { Better classification of emotional faces in the right } \\
\text { than the left visual field during unp odor exposure. } \\
\text { Participants faster at recognizing faces in left visual } \\
\text { field during exposure to odors in right nostril. }\end{array}$ \\
\hline
\end{tabular}

2 Unp $=$ unpleasant; ple $=$ pleasant; neu $=$ neutral; pos $=$ positive/positively; neg $=$

3 negative/negatively; $\exp =$ experiment; val = valence/valenced.

4

5 Discussion

6 The overall pattern emerging from the reviewed studies suggests that valenced odors indeed

7 affect face evaluations. The most consistent finding is that valenced odors affect evaluations in

8 general (e.g., all faces are perceived as more unpleasant in an unpleasant odor condition). This

9 indicates that a valenced odor might change the participants' emotional state, which biases their

10 evaluation of faces, or that when making valence evaluations of a face, participants misattribute

11 the source of the being evaluated. A few studies show odor-face congruency effects on

12 evaluations (i.e., that the combination of an odor eliciting an emotion affects the processing of

13 faces expressing specifically that emotion). In emotion classification tasks, a few of the studies 
1 found evidence for odor and facial expression integration. However, the majority of the findings

2 indicated enhanced response latencies in odor conditions relative to no-odor control conditions.

3 The experimental paradigms used can be divided into two categories; first, effects of valenced

4 odors, and second, classical conditioning with valenced odors, such that the valenced odor effect appears via a previously established association to the face stimulus. The results are reviewed in more detail below.

\section{Odor effects on the processing of face stimuli}

In this review, nine studies used response times as a measure to investigate the effects of valenced odors on emotion recognition. (Leppanen \& Hietanen, 2003) were the first to show that pleasant odors facilitated the classification accuracy of happy faces. In contrast, the speed of classifying disgusted faces was not affected by either pleasant or unpleasant odors. In the same study, a second experiment confirmed the effect of pleasant odors on happy faces. Although several studies have used similar methods as Leppanen and Hietanen (2003), this study's odor-face congruency effects have not yet been replicated. Instead, in a similar experimental design, Li, Jia, and Wang (2020) found that facial expressions (happy, neutral, and fearful) were overall recognized faster in an unpleasant food odor context. This effect was most pronounced for fearful faces. The effect was not present for happy faces in the pleasant odor condition. In two separate studies by Seubert and colleagues, the results showed only an overall effect of odor presence on improving face classification (Seubert, Kellermann, et al., 2010; Seubert, Loughead, et al., 2010). In one of these studies, participants were more accurate and faster in recognizing disgusted expressions in both the pleasant and unpleasant odor conditions and slower at recognizing happy faces than in the neutral condition (Seubert, Kellermann, et al., 2010). In the second study, the results were similar, but this time only present for disgusted faces (Seubert, Loughead, et al., 2010). Hence, these results are difficult to consolidate. One reason for the heterogeneity of results might be that performance is already close to the ceiling level in a simple task such as emotion classification. If odor effects are generally relatively small, there is not much room for improvement. The results of (Leleu et al., 2015) hints at this possibility. In this study, emotional expressions were morphed with neutral faces to show various levels of emotional intensity. The results demonstrated that facial expressions morphed with neutral faces were recognized at lower levels in congruent odor conditions (e.g., happy faces in the pleasant odor condition), for happy, disgusted, and angry facial expressions (Leleu et al., 2015). However, the results in a recent study by (Syrjänen, Liuzza, Fischer, \& Olofsson, 2017) showed that the recognition of dynamically morphing 
1 happy or disgusted faces was not affected by congruent odors. In the latter study, exploratory 2 results indicated that happy and disgusted expressions were recognized faster during exposure 3 to an unpleasant odor. Some studies have also presented stimuli in the left and right visual 4 fields. In (Stankovic, Nesic, \& Milic, 2020), participants performed an emotion classification task on neutral or happy, surprised, fearful, angry, disgusted, and sad facial expressions. Pictures of faces were presented in the left or right visual fields while participants were exposed to an unpleasant odor in the left or right nostril. Participants were faster at recognizing faces overall in the left visual field (right hemisphere) during exposure to the odor in the right nostril (right hemisphere), indicating a cross-modal confluence in the right hemisphere resulted in a processing speed advantage; however, the study had no neutral control condition, so it is possible that the arousing effect of the odor, irrespective of its valence, improved processing of faces in the stimulated hemisphere (Stankovic et al., 2020). The right-hemisphere effect is consistent with results from our team that the face-sensitive N170 ERP component is more prominent in the right hemisphere; furthermore, our study showed that the responsivity of the N170 to disgusted faces is enhanced by an unpleasant odor (Syrjänen et al., 2018). Another recent study investigated whether odors might direct attention toward odor-congruent happy and disgusted facial expressions in a dot-probe task (Syrjänen, Fischer, \& Olofsson, 2019). However, this study's results gave no evidence that attention was automatically directed towards odor-congruent facial expressions. However, the results instead indicated that probes in the unpleasant odor condition were uniquely detected more rapidly over repeated trials within a block, raising the possibility that visual processing benefits of malodor exposure might accumulate over time. Similar time-on-task effects were found by (Damjanovic, Wilkinson, \& Lloyd, 2018) in a visual search task. The authors found that happy faces were recognized faster initially in a pleasant and an unpleasant odor context; however, this effect was reversed toward the end of the task.

While there is some evidence to suggest valenced odors might improve visual processing of emotionally congruent facial expressions, especially the combination of unpleasant odor and disgusted or fearful faces, the evidence is mixed. Increasing task difficulty employing brief face duration times, subtle or morphed expressions, etc., might be needed to achieve consistent effects of background odors. There are also some indications that odor effects develop as a function of exposure time during the experiment. Not accounting for such effects might explain some of the mixed findings. 
Odor effects on evaluations of faces

2 Ten studies assessed how valenced odors affect the subjective properties of faces. Two of these studies showed that neutral faces were rated as more unpleasant in the context of an unpleasant odor (Cook et al., 2015; Cook et al., 2018) and more pleasant during a pleasant odor context (Cook et al., 2015). However, one study did not find any effect of pleasant odors on classifying neutral faces as either pleasant or unpleasant, which might indicate that binary classifications of faces are less sensitive than ratings to the presumably subtle influence of odors (Bensafi, Pierson, et al., 2002). Several studies have shown that faces, regardless of expression, are rated as more arousing and that the face valence is affected in the direction of the odor valence (Syrjänen et al., 2019; Syrjänen et al., 2018). However, results are not entirely consistent in this literature. Using Bayesian statistics, Syrjänen et al. (2017) found evidence against odor effects in face evaluations.

Ratings are also used to study valence congruency effects. One study showed congruency effects between facial expressions and odor valence; faces displaying happiness were rated as more pleasant during exposure to a pleasant odor, and disgusted faces were rated as more unpleasant during exposure to unpleasant odor (Cook et al., 2017). Odors may also affect facial attractiveness, a construct closely related to pleasantness; in one study, faces were rated as younger and more attractive-looking in a pleasant odor context (Seubert, Gregory, Chamberland, Dessirier, \& Lundstrom, 2014). In a similar vein, Dematte, Osterbauer, and Spence (2007) found that faces were rated as less attractive in the presence of an unpleasant odor. However, Cann and Ross (1989) found no effects of pleasant and unpleasant odors on attractiveness evaluations in a blocked design. Finally, one study indicated that such facial evaluations were affected by odor exposure at a subthreshold level. Here Li, Moallem, Paller, and Gottfried (2007) found that only participants unaware of the presence of unpleasant odor were influenced to rate neutral faces as less likable. However, the evidence reviewed above suggests that also readily perceived odors may have similar influences on face evaluations.

\section{Olfactory conditioning: learned odor-face associations}

In conditioning paradigms, associations between stimuli are created by simultaneous presentations. Here, we focus on olfactory conditioning studies that use face and odor stimuli. In studies on this topic, an unpleasant odor (unconditioned stimuli, UCS) is usually associated with a face $(\mathrm{CS}+)$ by presenting these concurrently; faces that are not associated with the UCS (CS-) are used as a control. Some studies have used odors as a contextual cue and instead used 
1 startle sounds (sudden loud noises) as UCS (Hermann, Ziegler, Birbaumer, \& Flor, 2000).

2 These designs allow studying how learned cross-modal associations shape the processing of

3 face stimuli; faces associated with unpleasant odors as CS+ may be processed differently from

4 the CS- associated faces. Two studies have shown response-time effects in a gender

5 classification task, but results are inconsistent; CS+ faces associated with an unpleasant

6 olfactory UCS were initially faster at the beginning of the task (Gottfried \& Dolan, 2004).

7 However, in another similar study (Gottfried, O'Doherty, \& Dolan, 2002), results showed

8 slowed responses to the CS+ faces conditioned with either pleasant or unpleasant UCS odors.

9 More research with high-powered samples is needed to establish how valenced odor 10 conditioning might modulate face classifications.

Several conditioning studies have shown similar results as when odors and faces are presented concurrently, faces previously conditioned by an unpleasant odor are evaluated more negatively (Hermann et al., 2000; Steinberg et al., 2013; Steinberg et al., 2012). Steinberg et al. (2013) found that faces conditioned with pleasant odors were preferred over faces paired with clean air and an unpleasant odor. In four experiments, Todrank, Byrnes, Wrzesniewski, and Rozin (1995) found that neutral faces associated with pleasant and unpleasant odors were liked and disliked, respectively. However, this effect was only consistent with odors that were people related (e.g., soap or sweat like odors), but not for object-related odors such as woody cedarlike odors. One study showed that faces were rated as more arousing if they were paired with either pleasant or unpleasant odors (Hermann et al., 2000). Thus, similarly to contextual odors, odor conditioning seems to have robust effects on facial evaluation, with odors biasing the evaluation of faces in the odor valence direction. This capacity is highlighted in a study by (Steinberg et al., 2012). The authors showed that in a stimulus set of 208 faces, only two CS+ pairings (neutral faces) with the unpleasant UCS odor were enough to affect facial evaluations negatively. We now know that animals can associate aversive odors with visual objects in a fast and flexible manner (Handler et al., 2019). These findings show a similar capacity in humans to rapidly associate faces with aversive odors.

\section{Future directions}

31 In the review above, we have highlighted key results emerging from the literature on odor effects on face perception. Several avenues remain to be explored in further research. For example, only a few studies investigated how interindividual variation in odor sensitivity affected odor effects in facial perception. Individuals vary considerably in their odor 
1 preferences, which might influence how different odors affect facial perception. For example,

2 people vary in disgust sensitivity (Tybur, Cinar, Karinen, \& Perone, 2018), which might be

3 especially relevant for how unpleasant odors might affect perception of facial expressions.

4 Here, a newly developed body odor disgust scale might be a suitable instrument for

5 investigating individual differences in olfactory related disgust (BODS; Liuzza, Lindholm, et

6 al., 2017). Although the BODS was developed for measuring body odor related disgust, it has

7 excellent construct validity in relation to existing disgust scales and has been shown to predict

8 disgust responses to human sweat bio-samples (Liuzza, Olofsson, Sabiniewicz, \& Sorokowska,

9 2017).

11 Nevertheless, individual variation is not only relevant for unpleasant odors. In our work, we 12 have observed that odors regarded as pleasant (e.g., lilac) might be perceived as unpleasant at 13 higher concentrations by some participants (Syrjänen, 2020; Syrjänen et al., 2018). Conversely, 14 an unpleasant odor such as valeric acid is by some participants rated as positively valenced (see 15 Figure 1 for a summary of valence ratings for these odors at three concentrations). Although 16 olfactory valence is sometimes thought of as a hard-wired response, perhaps universal 17 (Yeshurun \& Sobel, 2010), our observations suggest this assumption may lead to an 18 underestimation of the individual variation in olfactory valence evaluations. Future work could 19 instead tailor specific odors for each participant to maximize valence effects. 


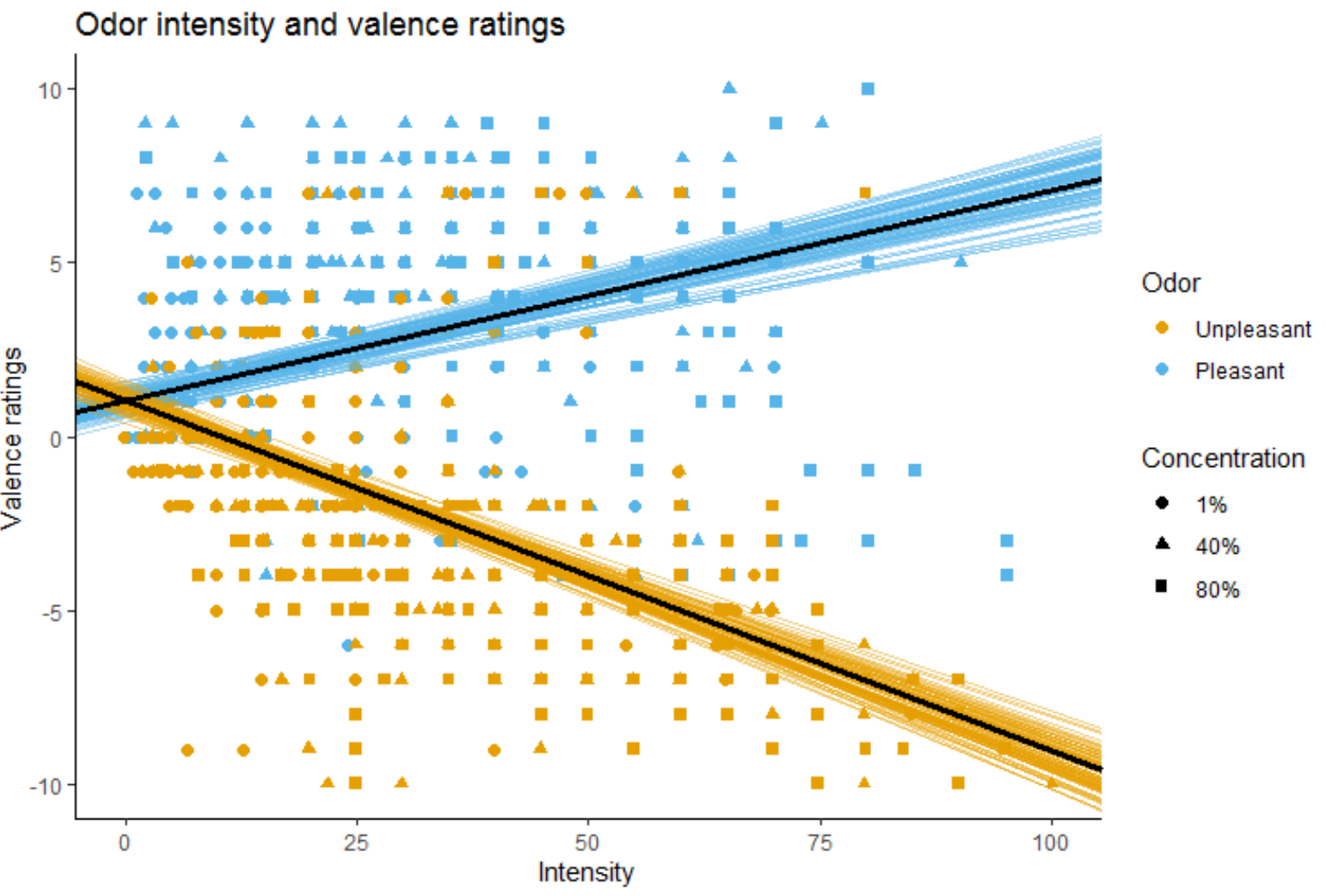

Figure 1. Rated odor intensity and valence for three different concentrations of valeric acid (unpleasant) and lilac essence (pleasant). The black lines show the predicted linear relationship between intensity and valence. Uncertainty of the linear effect (colored lines) is visualized with a sample of draws from the posterior distribution. Figure reprinted with permission from the author.

6 Among the reviewed studies, many have used unpleasant, presumably disgusting odors and 7 facial expressions such as angry or fearful that do not have a natural connection to these odors.

8 This usage might reflect the fact that anger and fear are well investigated in affect perception research but that these emotions are not easily mapped onto the affective space of odors.

10 Instead, we argue that the basic emotion most well-matched by unpleasant odors is disgust, and 11 for pleasant odors, happiness (Bensafi, Rouby, et al., 2002; Croy et al., 2011; Glass et al., 2014).

12 Future research should consider odors congruent with distinct emotional expressions, and this 13 olfactory-visual congruency should be empirically validated. For example, odors such as smoke 14 may elicit fear in specific contexts (Stevenson, 2010). Thus, the odor might modulate the processing of fearful expressions in a context-dependent way. Future studies might also explore 16 the connection between odors and mood and how these relate to the perception of emotional 17 expressions. Also, interindividual factors such as gender, age, and culture should be 18 investigated in future research. These factors typically affect the perception of emotional 19 information in other sensory modalities such as vision and hearing (Cortes, Laukka, Ebner, \& 
1 Fischer, 2019; Cortes, Laukka, Lindahl, \& Fischer, 2017) and therefore probably also in the

It is important to note that the studies reviewed here are not characterized by open research practices such as transparent and preregistered study protocols and open data. In recent years there has been an open debate within psychology due to the low reproducibility of research findings (Open Science Collaboration, 2015). Transparent and open study protocols might also reduce practices that result in non-replicable findings such as hypothesizing after results are known (HARKing; Kerr, 1998). Considering that at least half of the studies utilized small sample sizes and that odor effects typically may be assumed to be subtle, we can expect that some of the findings in this area are false positives or negatives (Button et al., 2013). Almost all studies found some effects of odor on facial perception. If we assume that many studies in the field are underpowered, there might be a file drawer problem of conducted but not published studies due to non-significant findings. The research in this area could also benefit from standardized measures, testing procedures, and stimuli. Practices that have been highly successful in emotion research include using standardized stimuli such as the international affective picture set (IAPS; Lang, Bradley, \& Cuthbert, 2008), and assessments such as the selfassessment manikin (SAM; Bradley \& Lang, 1994). Standardization would benefit the research community by making it easier to compare results across studies and sustain an incremental research endeavor.

Because of the relatively small number of published studies to date, open data would be beneficial for the whole community of researchers. Open data practices will enable reinterpretation and creation of novel hypotheses using existing data, and such practices will reduce the need for duplications. On a positive note, the recent response from the olfactory research community (www.gcchemosensr.org) with a large multinational study on olfactory related changes in COVID-19 infection (Parma et al., 2020) might in the future be used as a platform for large scale replication efforts.

\section{Conclusions}

We reviewed studies that span 25 years of olfactory research on odor effects on facial perception. Generally, the results indicate that facial expressions are classified more rapidly in the context of odors. Apart from a few studies that show face-odor valence congruency effects, 
1 the most consistent finding is that valenced odors affect perceived face valence overall (e.g.,

2 faces are perceived as more unpleasant in an unpleasant odor condition). Rapid classification

3 of faces is often unaffected by the congruency of face-odor pairings. However, there is some

4 evidence that an odor's presence improves the response speed due to overall arousal effects.

5 Establishing important individual differences markers and how odor effects may accumulate as

6 a function of exposure time remain questions of importance for future work. More work on

7 standardizing stimuli and experimental protocols and adopting open research practices may

8 increase the robustness of results and might help address outstanding questions in this field.

\section{Acknowledgments}

11 This research was funded by the Swedish Research Council (2016-02018)

12

\section{References}

Alaoui-Ismaïli, O., Robin, O., Rada, H., Dittmar, A., \& Vernet-Maury, E. (1997). Basic Emotions Evoked by Odorants. Physiology and Behavior, 62(4), 713-720. doi:10.1016/s00319384(97)90016-0

Amsellem, S., Hochenberger, R., \& Ohla, K. (2018). Visual-olfactory Interactions: Bimodal Facilitation and Impact on the Subjective Experience. Chemical Senses, 43(5), 329-339. doi:10.1093/chemse/bjy018

Anderson, A. K., Christoff, K., Stappen, I., Panitz, D., Ghahremani, D. G., Glover, G., . . Sobel, N. (2003). Dissociated neural representations of intensity and valence in human olfaction. Nature Neuroscience, 6(2), 196-202. doi:10.1038/nn1001

Aviezer, H., Ensenberg, N., \& Hassin, R. R. (2017). The inherently contextualized nature of facial emotion perception. Curr Opin Psychol, 17, 47-54. doi:10.1016/j.copsyc.2017.06.006

Barrett, L. F. (2017a). How Emotions Are Made: The Secret Life of the Brain. New York: Houghton Mifflin Harcourt. 
Barrett, L. F. (2017b). The theory of constructed emotion: an active inference account of interoception and categorization. Social Cognitive and Affective Neuroscience, 12(1), 1-23. doi:10.1093/scan/nsw154

Barrett, L. F., Adolphs, R., Marsella, S., Martinez, A. M., \& Pollak, S. D. (2019). Emotional Expressions Reconsidered: Challenges to Inferring Emotion From Human Facial Movements. Psychological Science in the Public Interest, 20(1), 1-68. doi:10.1177/1529100619832930

Bensafi, M. (2002). Autonomic Nervous System Responses to Odours: the Role of Pleasantness and Arousal. Chemical Senses, 27(8), 703-709. doi:10.1093/chemse/27.8.703

Bensafi, M., Pierson, A., Rouby, C., Farget, V., Bertrand, B., Vigouroux, M., . . Holley, A. (2002). Modulation of visual event-related potentials by emotional olfactory stimuli. Neurophysiologie Clinique/Clinical Neurophysiology, 32(6), 335-342. doi:10.1016/s0987-7053(02)00337-4

Bensafi, M., Rouby, C., Farget, V., Bertrand, B., Vigouroux, M., \& Holley, A. (2002). Psychophysiological correlates of affects in human olfaction. Neurophysiologie Clinique/Clinical Neurophysiology, 32(5), 326-332. doi:10.1016/s0987-7053(02)00339-8

Bradley, M. M., \& Lang, P. J. (1994). Measuring emotion: The self-assessment manikin and the semantic differential. Journal of Behavior Therapy and Experimental Psychiatry, 25(1), 49-59. doi:10.1016/0005-7916(94)90063-9

Button, K. S., Ioannidis, J. P., Mokrysz, C., Nosek, B. A., Flint, J., Robinson, E. S., \& Munafo, M. R. (2013). Power failure: why small sample size undermines the reliability of neuroscience. Nature Reviews: Neuroscience, 14(5), 365-376. doi:10.1038/nrn3475

Cann, A., \& Ross, D. A. (1989). Olfactory Stimuli as Context Cues in Human Memory. The American Journal of Psychology, 102(1). doi:10.2307/1423118

Cook, S., Fallon, N., Wright, H., Thomas, A., Giesbrecht, T., Field, M., \& Stancak, A. (2015). Pleasant and Unpleasant Odors Influence Hedonic Evaluations of Human Faces: An Event-Related Potential Study. Frontiers in Human Neuroscience, 9, 661. doi:10.3389/fnhum.2015.00661

Cook, S., Kokmotou, K., Soto, V., Fallon, N., Tyson-Carr, J., Thomas, A., . . Stancak, A. (2017). Pleasant and unpleasant odour-face combinations influence face and odour perception: An event-related potential study. Behavioural Brain Research, 333, 304-313. doi:10.1016/j.bbr.2017.07.010

Cook, S., Kokmotou, K., Soto, V., Wright, H., Fallon, N., Thomas, A., . . Stancak, A. (2018). Simultaneous odour-face presentation strengthens hedonic evaluations and event-related potential responses influenced by unpleasant odour. Neuroscience Letters, 672, 22-27. doi:10.1016/j.neulet.2018.02.032

Cortes, D. S., Laukka, P., Ebner, N. C., \& Fischer, H. (2019). Age-related differences in evaluation of social attributes from computer-generated faces of varying intensity. Psychology and Aging, 34(5), 686-697. doi:10.1037/pag0000364 
Cortes, D. S., Laukka, P., Lindahl, C., \& Fischer, H. (2017). Memory for faces and voices varies as a function of sex and expressed emotion. PloS One, 12(6), e0178423. doi:10.1371/journal.pone. 0178423

Croy, I., Olgun, S., \& Joraschky, P. (2011). Basic emotions elicited by odors and pictures. Emotion, 11(6), 1331-1335. doi:10.1037/a0024437

Damjanovic, L., Wilkinson, H., \& Lloyd, J. (2018). Sweet Emotion: The Role of Odor-induced Context in the Search Advantage for Happy Facial Expressions. Chemical Senses, 43(3), 139-150.

de Vries, R., Morquecho-Campos, P., de Vet, E., de Rijk, M., Postma, E., de Graaf, K., . . Boesveldt, S. (2020). Human spatial memory implicitly prioritizes high-calorie foods. Scientific Reports, 10(1), 15174. doi:10.1038/s41598-020-72570-x

Dematte, M. L., Osterbauer, R., \& Spence, C. (2007). Olfactory cues modulate facial attractiveness. Chemical Senses, 32(6), 603-610. doi:10.1093/chemse/bjm030

Gottfried, J. A., O'Doherty, J., \& Dolan, R. J. (2002). Appetitive and aversive olfactory learning in humans studied using event-related functional magnetic resonance imaging. The Journal of Neuroscience, 22(24), 10829-10837.

Handler, A., Graham, T. G. W., Cohn, R., Morantte, I., Siliciano, A. F., Zeng, J., . . Ruta, V. (2019). Distinct Dopamine Receptor Pathways Underlie the Temporal Sensitivity of Associative Learning. Cell, 178(1), 60-75 e19. doi:10.1016/j.cell.2019.05.040 
Hermann, C., Ziegler, S., Birbaumer, N., \& Flor, H. (2000). Pavlovian aversive and appetitive odor conditioning in humans: subjective, peripheral, and electrocortical changes. Experimental Brain Research, 132(2), 203-215. doi:10.1007/s002210000343

Herz, R. S. (2009). Aromatherapy facts and fictions: a scientific analysis of olfactory effects on mood, physiology and behavior. International Journal of Neuroscience, 119(2), 263-290. doi:10.1080/00207450802333953

Hummel, T., Sekinger, B., Wolf, S. R., Pauli, E., \& Kobal, G. (1997). 'Sniffin' Sticks': Olfactory performance assessed by the combined testing of odor identification, odor discrimination and olfactory threshold. Chemical Senses, 22(1), 39-52. doi:10.1093/chemse/22.1.39

Kerr, N. L. (1998). HARKing: hypothesizing after the results are known. Personality and Social Psychology Review, 2(3), 196-217. doi:10.1207/s15327957pspr0203_4

Lang, P. J., Bradley, M. M., \& Cuthbert, B. N. (2008). International affective picture system (IAPS): Affective ratings of pictures and instruction manual. Technical report A-8.

Leleu, A., Demily, C., Franck, N., Durand, K., Schaal, B., \& Baudouin, J. Y. (2015). The Odor Context Facilitates the Perception of Low-Intensity Facial Expressions of Emotion. PloS One, 10(9), e0138656. doi:10.1371/journal.pone.0138656

Leppanen, J. M., \& Hietanen, J. K. (2003). Affect and face perception: odors modulate the recognition advantage of happy faces. Emotion, 3(4), 315-326. doi:10.1037/1528-3542.3.4.315

Li, D., Jia, J., \& Wang, X. (2020). Unpleasant Food Odors Modulate the Processing of Facial Expressions: An Event-Related Potential Study. Frontiers in Neuroscience, 14. doi: $10.3389 /$ fnins. 2020.00686

Li, W., Moallem, I., Paller, K. A., \& Gottfried, J. A. (2007). Subliminal smells can guide social preferences. Psychological Science, 18(12), 1044-1049. doi:10.1111/j.14679280.2007.02023.x

Lindquist, K. A., Wager, T. D., Kober, H., Bliss-Moreau, E., \& Barrett, L. F. (2012). The brain basis of emotion: a meta-analytic review. Behavioral and Brain Sciences, 35(3), 121-143. doi:10.1017/S0140525X11000446

Liuzza, M. T., Lindholm, T., Hawley, C., Senden, M. G., Ekstrom, I., Olsson, M. J., . . Olofsson, J. K. (2017). The Body Odor Disgust Scale (BODS): Development and Validation of a Novel Olfactory Disgust Assessment. Chemical Senses, 42(6), 499-508. doi:10.1093/chemse/bjw107

Liuzza, M. T., Olofsson, J. K., Sabiniewicz, A., \& Sorokowska, A. (2017). Body odor trait disgust sensitivity predicts perception of sweat bio-samples. Chemical Senses. doi:10.1093/chemse/bjx026

McGann, J. P. (2017). Poor human olfaction is a 19th-century myth. Science, 356(6338). doi:10.1126/science.aam7263

Millot, J.-L., Brand, G., \& Morand, N. (2002). Effects of ambient odors on reaction time in humans. Neuroscience Letters, 322(2), 79-82. doi:10.1016/s0304-3940(02)00092-7 
Olofsson, J. K., \& Gottfried, J. A. (2015). The muted sense: neurocognitive limitations of olfactory language. Trends in Cognitive Sciences, 19(6), 314-321. doi:10.1016/j.tics.2015.04.007

Open Science Collaboration. (2015). Estimating the reproducibility of psychological science. Science, 349(6251), aac4716. doi:10.1126/science.aac4716

Parma, V., Ohla, K., Veldhuizen, M. G., Niv, M. Y., Kelly, C. E., Bakke, A. J., . . Hayes, J. E. (2020). More Than Smell-COVID-19 Is Associated With Severe Impairment of Smell, Taste, and Chemesthesis. Chemical Senses, 45(7), 609-622. doi:10.1093/chemse/bjaa041

Ross, L. A., Saint-Amour, D., Leavitt, V. M., Javitt, D. C., \& Foxe, J. J. (2007). Do you see what I am

Russell, J. A. (1993). Forced-choice response format in the study of facial expression. Motivation and Emotion, 17(1), 41-51. doi:10.1007/bf00995206

Seo, H. S., Roidl, E., Muller, F., \& Negoias, S. (2010). Odors enhance visual attention to congruent objects. Appetite, 54(3), 544-549. doi:10.1016/j.appet.2010.02.011

Seubert, J., Gregory, K. M., Chamberland, J., Dessirier, J. M., \& Lundstrom, J. N. (2014). Odor valence linearly modulates attractiveness, but not age assessment, of invariant facial features in a memory-based rating task. PloS One, 9(5), e98347. doi:10.1371/journal.pone.0098347

Seubert, J., Kellermann, T., Loughead, J., Boers, F., Brensinger, C., Schneider, F., \& Habel, U. (2010). Processing of disgusted faces is facilitated by odor primes: a functional MRI study. Neuroimage, 53(2), 746-756. doi:10.1016/j.neuroimage.2010.07.012

Seubert, J., Loughead, J., Kellermann, T., Boers, F., Brensinger, C. M., \& Habel, U. (2010). Multisensory integration of emotionally valenced olfactory-visual information in patients with schizophrenia and healthy controls. Journal of Psychiatry and Neuroscience, 35(3), 185-194. doi:10.1503/jpn.090094

Soudry, Y., Lemogne, C., Malinvaud, D., Consoli, S. M., \& Bonfils, P. (2011). Olfactory system and emotion: common substrates. European Annals of Otorhinolaryngology, Head and Neck Diseases, 128(1), 18-23. doi:10.1016/j.anorl.2010.09.007

Stankovic, M., Nesic, M., \& Milic, J. (2020). Effects of unpleasant odors on emotion recognition: The right hemisphere and valence-specific hypotheses. Psihologija, 53(2), 183-198. doi:10.2298/psi190704019s

Steinberg, C., Brockelmann, A. K., Dobel, C., Elling, L., Zwanzger, P., Pantev, C., \& Junghofer, M. (2013). Preferential responses to extinguished face stimuli are preserved in frontal and occipitotemporal cortex at initial but not later stages of processing. Psychophysiology, 50(3), 230-239. doi:10.1111/psyp. 12005

Steinberg, C., Dobel, C., Schupp, H. T., Kissler, J., Elling, L., Pantev, C., \& Junghofer, M. (2012). Rapid and highly resolving: affective evaluation of olfactorily conditioned faces. Journal of Cognitive Neuroscience, 24(1), 17-27.doi:10.1162/jocn_a_00067 
Stevenson, R. J. (2010). An initial evaluation of the functions of human olfaction. Chemical Senses, 35(1), 3-20. doi:10.1093/chemse/bjp083

Syrjänen, E. (2020). The effects of valenced odors on facial perception. (PhD), Stockholm University, Stockholm.

Syrjänen, E., Fischer, H., \& Olofsson, J. K. (2019). Background odors affect behavior in a dot-probe task with emotionally expressive faces. Physiology and Behavior. doi:10.1016/j.physbeh.2019.05.001

Syrjänen, E., Liuzza, M. T., Fischer, H., \& Olofsson, J. K. (2017). Do Valenced Odors and Trait Body Odor Disgust Affect Evaluation of Emotion in Dynamic Faces? Perception, 301006617720831. doi:10.1177/0301006617720831

Syrjänen, E., Wiens, S., Fischer, H., Zakrzewska, M., Wartel, A., Larsson, M., \& Olofsson, J. K. (2018). Background Odors Modulate N170 ERP Component and Perception of Emotional Facial Stimuli. Frontiers in Psychology, 9, 1000. doi:10.3389/fpsyg.2018.01000

Todrank, J., Byrnes, D., Wrzesniewski, A., \& Rozin, P. (1995). Odors Can Change Preferences for People in Photographs - a Cross-Modal Evaluative Conditioning Study with Olfactory Uss and Visual Css. Learning and Motivation, 26(2), 116-140. doi:Doi 10.1016/0023-9690(95)90001-2

Tybur, J. M., Cinar, C., Karinen, A. K., \& Perone, P. (2018). Why do people vary in disgust? Philosophical Transactions of the Royal Society of London. Series B: Biological Sciences, 373(1751). doi:10.1098/rstb.2017.0204

Wieser, M. J., \& Brosch, T. (2012). Faces in context: a review and systematization of contextual influences on affective face processing. Frontiers in Psychology, 3, 471. doi:10.3389/fpsyg.2012.00471

Yeshurun, Y., \& Sobel, N. (2010). An odor is not worth a thousand words: from multidimensional odors to unidimensional odor objects. Annual Review of Psychology, 61, 219-241, C211-215. doi:10.1146/annurev.psych.60.110707.163639 